status I and presented less often with adverse prognostic factors such as low serum albu$\min (<35 \mathrm{~g} / \mathrm{l} ; 39 \%$ vs $26 \%$ ), low hemoglobin (<10 g/dl; 33\% vs 41\%), or elevated C-reactive protein levels ( $\geq 2 \mathrm{mg} / \mathrm{dl}$; $24 \%$ vs $29 \%$ ). Survival (after adjusting for age-related differences in survival expectancy) was significantly longer in the younger cohort than in the older cohort after both conventional and high-dose therapy (median survival for conventional therapy 4.5 years vs 3.3 years, $P<0.001$, and median survival for high-dose therapy 7.5 years vs 5.7 years, $P=0.04$ ). Multivariate analysis revealed that older age was an independent risk factor for shortened survival in conventional therapy but not in high-dose therapy.

The authors suggest that the significantly longer survival of young patients with multiple myeloma might be attributable to the fact that they tend to present with more-favorable prognostic factors.

Original article Ludwig $\mathrm{H}$ et al. (2008) Myeloma in patients younger than age 50 years presents with more favorable features and shows better survival: an analysis of 10,549 patients from the International Myeloma Working Group. Blood 111: 4039-4047

\section{No survival benefit in follicular lymphoma with first-line intensive chemoimmunotherapy}

Although intensive chemoimmunotherapy regimens with autologous stem-cell transplantation improve survival in patients with follicular lymphoma who have relapsed, the efficacy of this therapy as a first-line treatment is less clear. Recent studies, however, have indicated that combining high-dose regimens with rituximab might prove effective in initial treatment.

A multicenter phase III trial has examined the outcomes of patients with high-risk follicular lymphoma who were randomized to first-line therapy with either rituximab-supplemented high-dose sequential chemotherapy and autologous stem-cell transplantation (R-HDSC; $n=68$ ) or conventional therapy (cyclosphosphamidedoxorubicin-vincristine-prednisone) followed by rituximab (CHOP-R; $n=66$ ).

The R-HDSC group had a better complete remission rate than the CHOP-R group (85\% vs $62 \% ; P<0.001)$; prospective 4 -year disease control was also better in patients treated with R-HDSC $(P<0.001)$. Overall survival, however, did not differ significantly between the treatment arms. Of the CHOP-R-assigned patients who relapsed or had refractory disease, overall survival at 30 months and projected 3-year outcomes were better in the individuals who underwent salvage therapy with R-HDSC. In multivariate analysis, molecular remission was the strongest independent predictor of outcome and might, therefore, have prognostic value.

The authors conclude that the disease control provided by first-line R-HDSC does not translate into improved overall survival in high-risk patients with follicular lymphoma and suggest that intensive chemoimmunotherapy might be best reserved for second-line therapy in these individuals.

Original article Ladetto M et al. (2008) Prospective, multicenter randomized GITMO/IIL trial comparing intensive (R-HDS) versus conventional (CHOP-R) chemoimmunotherapy in high-risk follicular lymphoma at diagnosis: the superior disease control of R-HDS does not translate into an overall survival advantage. Blood 111: 4004-4013

\section{High lymph-node yield improves the prognostic value of esophageal cancer staging}

The current International Union Against Cancer tumor-node-metastasis (TNM) classification system recommends that only six nodes need to be sampled in order to determine the nodal status of patients with esophageal carcinoma. Bogoevski et al. have found that in patients with carcinoma of the esophagus who have undergone curative resection, the otherwise poor prognostic value of lymph-node staging is improved when a high number of nodes are sampled or if the ratio of positive nodes to sampled nodes is low.

Of the 235 patients analyzed, 26 had a lymph-node yield (LNY) of $\leq 5,96$ had a LNY of 6-18 and 113 had a LNY of $\geq 19$. In patients with no nodal involvement ( $\mathrm{pNO}$ ), median survival was higher in those who had $\geq 19$ nodes sampled than in those who had $6-18$ or $\leq 5$ nodes sampled $(P=0.003$ and $P=0.05$, respectively). The number of nodes dissected had no significant effect on survival in patients with lymph-node involvement (pN1); however, individuals with a low ratio of positive lymph nodes to overall LNY had significantly better survival than did those with a higher ratio. 